\title{
Development of intelligent healthcare system based on ambulatory blood pressure measuring device
}

\author{
Sung-Jong Eun ${ }^{1} \cdot$ JungYoon $\mathrm{Kim}^{2}$ (B)
}

Received: 3 April 2020 / Accepted: 5 June 2020/Published online: 2 July 2020

(C) The Author(s) 2020

\begin{abstract}
Currently, the market size of blood pressure monitors both in domestic and overseas is gradually increasing due to the increase in hypertension patients resulting from aging population. In addition, the necessity of developing systems and devices for the healthcare of hypertension patients is also increasing. Moreover, the determination of health normality in respect to the management of hypertension patients is possible, but it is essentially important to incorporate preventive healthcare. Thus, further studies on deep learning-based prediction technology using previous data are needed. This paper proposes the development of an intelligent healthcare management system that can help to manage the health of hypertensive patients. The system includes a wrist-worn ambulatory blood pressure monitoring device that can analyze the normality of measured blood pressures. The performance evaluation results of the proposed system verified the reliability of data acquisition as compared with the existing equipment as well as the efficiency of the intelligent healthcare system.
\end{abstract}

Keywords Intelligent healthcare system · Ambulatory blood pressure monitoring · TOAST classification .

Signal similarity calculation

\section{Introduction}

In the USA, the world's largest medical device market, the blood pressure monitor market is continuously growing as the need for efficient and accurate blood pressure measurements increases due to the growth on the number of hypertension patients caused by the aging population. It is indicated that the market size amounted to U\$ 973 million with an increase of $10.8 \%$ over the previous year [1-3].

JungYoon Kim

kjyoon@gachon.ac.kr

Sung-Jong Eun

asclephios@naver.com

1 Health IT Research Center, Gil Medical Center, Gachon University College of Medicine, Incheon, Republic of Korea

2 Graduate School of Game, Gachon University, Sujung-Gu, Seongnam, Gyunggi-Do, Republic of Korea
The occurrence of hypertension is expected to increase due to limited exercise activities and the aging of baby boomers [4]. In addition, the implementation of programs for the prevention and management of adult diseases at the government level will also increase the demand for blood pressure monitors. The primary consumers will be hypertension patients, baby boomers, and medical service institutions (i.e., hospitals, government facilities, nursing homes, etc.). The unveiling of such devices that are easy to use, minimizes discomfort, efficiently and accurately measures the blood pressure and stimulates consumer demand.

The reliability of a professional blood pressure monitor used in medical institutions is essentially important; thus, higher measurement accuracy has a significant influence on the purchase decisions. With the development of technology, the simple and small size design allows easy and portable handling at home, creating new demand, and in fact, many automatic blood pressure monitors are widely available resulting in a large-scale blood pressure monitor 
market. However, the availability of ambulatory blood pressure monitoring devices that is able to measure fragmentary blood pressure has more advantages in managing hypertension since it provides information on daily blood pressure fluctuation patterns which are limited in the market due to its inconvenience and less portability. This minimal market for blood pressure monitoring devices leads to meager investments in device development. The ambulatory blood pressure monitoring devices were not yet universalized and are merely used for research purposes. If such devices will be developed to provide higher efficiency and accuracy, minimize discomfort and increase convenience, the paradigm of blood pressure management can be changed from the existing concept of hypertension. It can also reduce costs in improving the efficiency of national health.

Ambulatory blood pressure monitoring has several advantages over fractional blood pressure measurement, which measures blood pressure several times during normal activities [5]. First, the white coat effect can distinguish an average person diagnosed with hypertension. Ambulatory blood pressure monitoring is useful in costeffective ratios because it can reduce unnecessary medical expenses by identifying white coat hypertension. Second, the amount of time the patient was exposed to high blood pressure can be determined. Many clinical evidences were reported that there were more severe damages to the target organ whenever the mean blood pressure for $24 \mathrm{~h}$ will be higher than the blood pressure measured in the hospital and the longer the amount of time to have a higher blood pressure than the constant blood pressure (i.e., blood pressure load). Third, the fluctuation pattern of blood pressure can be determined which can be used to more efficiently treat hypertension. It is known that daytime blood pressure alone cannot predict the degree of nighttime blood pressure drop, and the non-dipper is observed in about $25 \%$ of hypertensive patients. Non-dipper hypertensions have been reported to have more complications such as left ventricular hypertrophy and asymptomatic stroke than the dipper hypertensions. Fourth, information about blood pressure fluctuations can be provided. The daily changes in blood pressure are more severe than with higher blood pressure, and the target organ damage is critical in people with sharp fluctuations in blood pressure regardless of the average blood pressure. Lastly, the evaluation of new blood pressure drugs can be advantageous. When evaluating new hypertension drugs, the effectiveness can be assessed in few patients through new indicators such as $T /$ $P$ ratios and effects on fluctuation patterns. Information on blood pressure variability is also provided to further characterize the new hypertension drugs.

Active measurement of ambulatory blood pressure monitoring should be undertaken, but the development of devices to perform such function is very slow. The current and widely used blood pressure meter measures the blood pressure through winding a cuff around the upper arm and inflated, which is too large and cumbersome to carry and gives a significant pain whenever the pressure is applied. In addition, pain can interfere with the patient's sound sleep when blood pressure is measured whenever the patient is asleep, specifically if the measurement is required every hour. Thereby, the reliability of blood pressure measured during sleep is lowered as well as patient compliance of measured blood pressure. Moreover, if the patient inevitably loosens the blood pressure cuff to take a shower or change clothes and then wears it again, reliability of the measured blood pressure can be lowered since it may not be worn again in an accurate manner. Thus, the necessity of an ambulatory blood pressure monitoring device having high portability, capable of minimizing patient discomfort, and with high reliability is essentially important. This paper aims to develop an efficient healthcare management system and design a device that can efficiently measure and manage the ambulatory blood pressure.

\section{Related works}

The reliability of professional blood pressure monitors used in medical institutions is considered as the most important factor that greatly influences the acquisition decision. With the development of technology, small size, simple usage, and convenient devices in homes are regularly being released, which creates new demand. Various automatic blood pressure monitors have been distributed creating a large-scale market for blood pressure monitors [6-10]. The ambulatory blood pressure monitoring devices can provide more advantages wherein it measures not only fragmentary blood pressures but can also manage high blood pressures through daily blood pressure fluctuation patterns. However, these devices provide usage inconvenience and were not portable such that its market is very limited, leading to a very low investment in its development. The previously developed ambulatory blood pressure monitoring devices are depicted in Table 1.

Many manufacturers are evaluating various measurement and analysis methods for the purpose of commercialization. However, most of the measuring devices were inconvenient to use, and even those good quality devices were insufficient in terms of the utilization of various information obtained by measuring ambulatory blood pressure. In addition, there are only few devices that were classified into the TOAST class which is the basis for the actual blood pressure analysis. Thus, this paper used more than 300 patient's data to learn and make clinical evidence of the TOAST group, used the extracted TOAST group 
Table 1 The existing ambulatory blood pressure monitoring devices

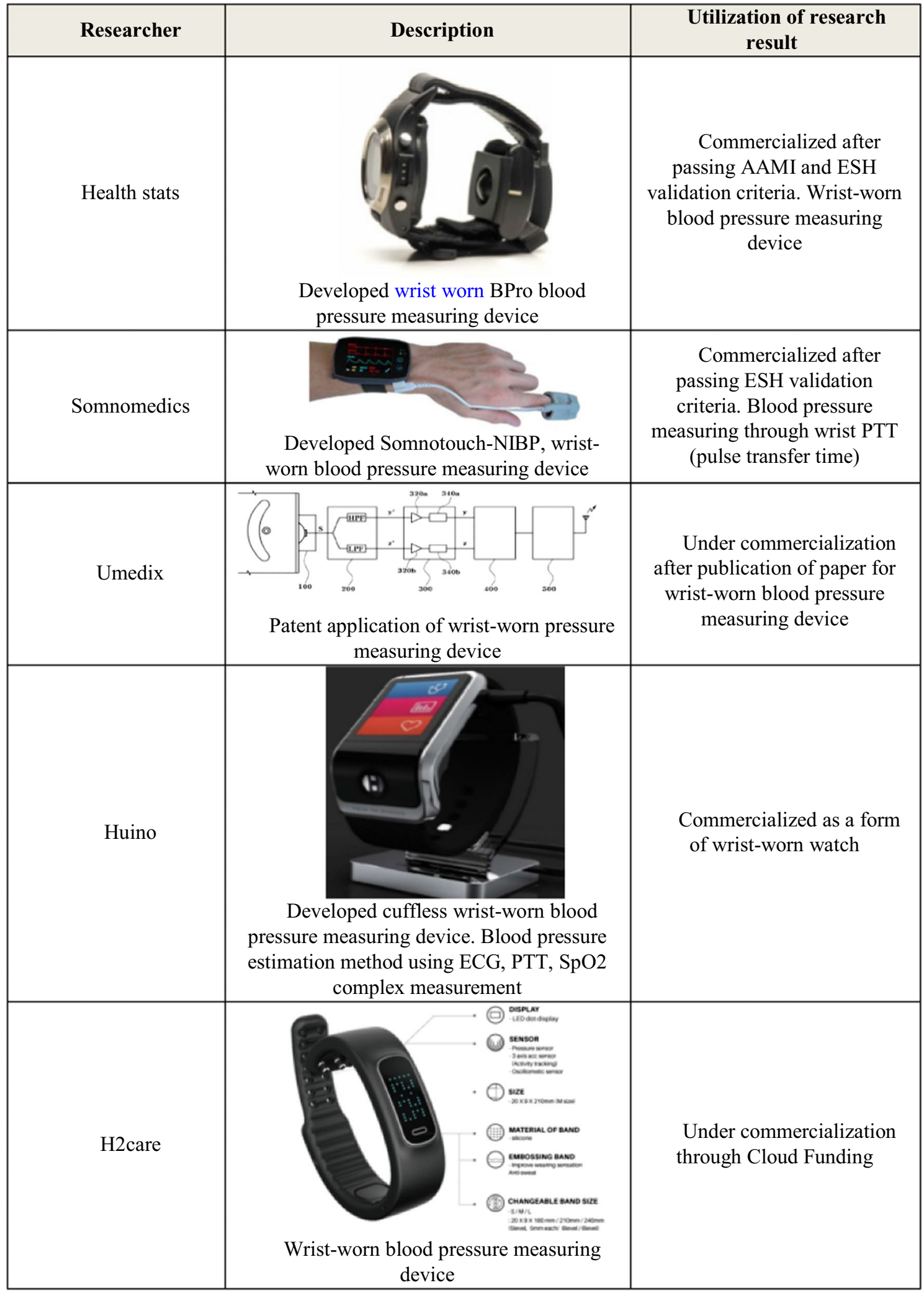


criteria as a guide, and reflected an evaluation process to distinguish normal or abnormal blood pressure measurements in order to develop an intelligent healthcare system based on ambulatory blood pressure monitoring device.

\section{Proposed intelligent healthcare management system}

The proposed intelligent healthcare management system based on ambulatory blood pressure monitor can be developed as shown in Fig. 1.

The hardware represents the actual manufactured ambulatory blood pressure monitoring device, and it can be managed by the user through interlocking with the PCbased analysis program using the Bluetooth module. Many intelligent analytic monitoring systems were being developed and embedded into wearable devices linked into back-end systems [11,12].

The proposed ambulatory blood pressure monitoring system requires the following conditions. First, the measuring device should be miniaturized so that it can be worn easily (e.g., on a wrist) and should be made of a material such as silicon for convenient wearing. Then, the system includes an algorithm that converts the gathered sensor signal into a measured blood pressure. In addition, blood pressure measurement is performed by both a control circuit module and an active pressure generator. The control circuit module controls the pressure so that it matches the intravascular pressure by contacting the pressure sensor to the blood pressure measuring area, while an active pressure generator changes the pressure continuously for blood pressure measurement. The portable ambulatory blood pressure monitoring device consists of a communication module that delivers measured information and a battery module that provides power to its components. The device is ultimately easy to use and provides a reliable and continuous blood pressure monitoring without pain. Moreover, in order to minimize the noise caused by external shocks, a fixed structure made of silicon that can closely adhere the pressure sensor to the wrist has been developed to compensate the noise caused by motion artifacts through a feedback algorithm by motor control.

Ultimately, a prototype was developed for a tonometertype wrist-worn ambulatory blood pressure monitoring device that is highly portable, easy to wear, and improves the reliability on the measurement signal. Furthermore, this paper proposed the development of an intelligent healthcare system that can continuously and conveniently manage the ambulatory blood pressure of users by mounting a TOAST program that may analyze the measured blood pressures.

\subsection{Development of a prototype for blood pressure monitoring device}

The ambulatory blood pressure monitor is designed as wrist band type for high portability and convenient wearing. The ring-shaped band can be worn on the user's wrist with an air pocket inside of the annular band that applies pressure. The ambulatory blood pressure of the user is measured by a pressure sensor that detects the air pressure inside the air pocket and measures the blood pressure waveform. The detailed components of the proposed ambulatory blood pressure monitoring device are depicted in Fig. 2.

The measurement of blood pressure can be represented in a five-step process. First, the blood pressure gauge is set near the user while keeping the band air pocket (i.e., first part) in contact with the user's wrist. Next, the air pressure is moved from the second part air pocket to the first part air pocket to increase the internal pressure on the first part air pocket which is attached on the user's wrist to enable the pressure sensor to measure the bio-signal. Then, the measured bio-signal waveforms are analyzed to derive an optimal value of the internal pressure of the first part air pocket. After that, the internal pressure on the first part air pocket is set to the optimum value. Finally, the blood pressure of the user is measured as a continuous waveform.
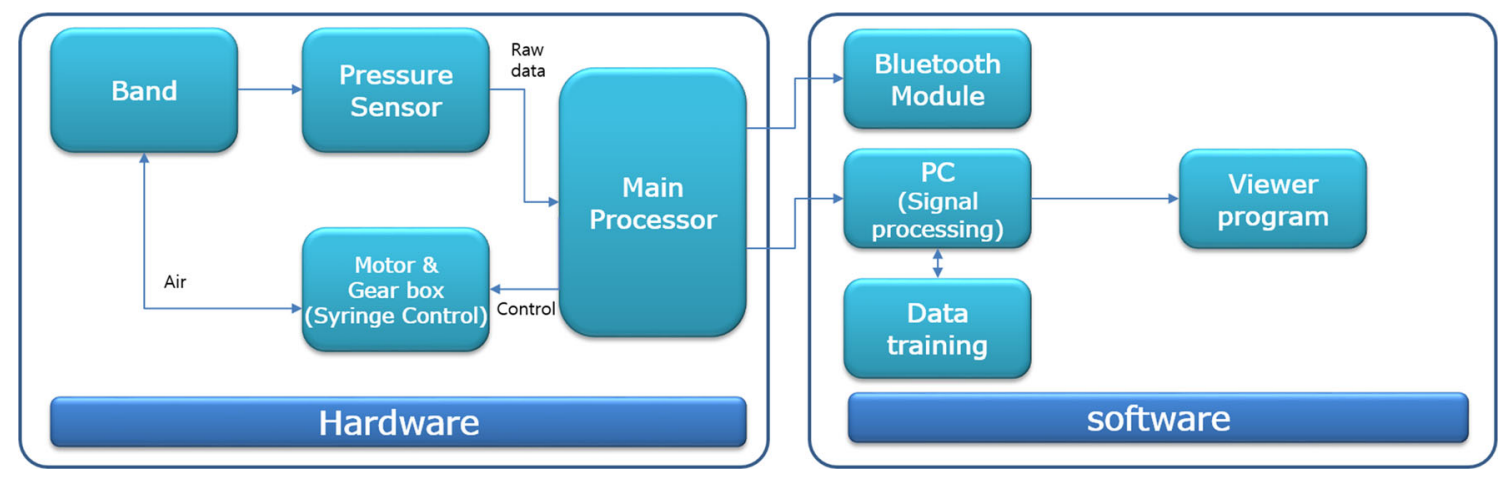

Fig. 1 Intelligent healthcare management system architecture 


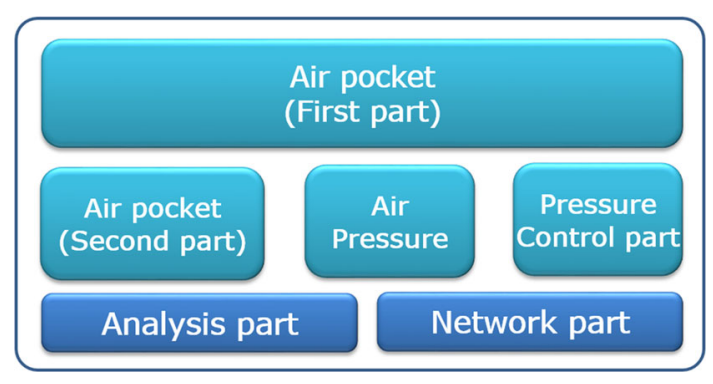

Fig. 2 Proposed blood pressure monitoring device structure

\subsubsection{Development of a tonometric 24-h ambulatory blood pressure monitoring system}

A pressure sensor driving circuit was developed to implement the wrist vein tonometric method using a tonometric pressure measurement module. The Arduino Nano v3.0 model was used as the main driver module for sensor data processing and wireless communication control. The Arduino Nano uses ATmega328 as its main processor and can support eight analog input and output ports and 22 digital input and output ports. The pressure sensor driving circuit considers an ADC resolution and motor driver module for close contact between pressure sensor and blood vessel. It was developed to connect a syringe to a driver motor by using a rack gear in order to inject or discharge syringe air pressure into the cuff (i.e., band air pocket). With the cuff positioned at the radial artery on the user's wrist, the band air pocket was inflated to expand the cuff, compressing the radial artery, and measuring the pressure change on the cuff through the beating of the radial artery.

\subsubsection{Development of an automatic feedback algorithm for motor control}

An error in the analysis increases as the cuff pressure changes due to the patient's movement; thus, an algorithm for estimating the optimal cuff pressure is developed to automatically control the driver motor so that the cuff pressure remains constant. The measured data by the pressure sensor are divided into pulse waves and cuff pressure using a digital filter, and the optimum cuff pressure is estimated by calculating the signal-to-noise ratio of the pulse waves according to the cuff pressure. The driver motor rotates to inject more air pressure when the measured cuff pressure is lower than the estimated value; otherwise, it rotates in reverse to release cuff pressure.

\subsubsection{Development of wireless data transmission}

The Arduino Nano board is used as the main controller module in the prototype, while the HC-06 Bluetooth module is for data wireless transmission. The HC-06 Bluetooth module has two separate pins used by the pressure sensor for the reception of the measured signals and its wireless transmission to the PC. Both low-pass and highpass digital filters were implemented to the measured biosignal to distinguish cuff pressure from pulse waves. In addition, the systolic, diastolic, mean blood pressure, and other cardiovascular parameters in the pulse wave were calculated.

Moreover, the radial artery beats were extracted during the filtering process to improve the accuracy of the blood pressure estimation algorithm using the wrist-worn pulse wave data as shown in Fig. 3. The slope of filtering mask and regression analysis were performed by comparing the extracted beats with the finger pressure waveform to increase the measurement accuracy.

\subsubsection{Development of prototype for ambulatory blood pressure monitoring device}

A $5 \times 3 \mathrm{~cm}$ cuff was designed using a wrist band fixing mechanism to be easily worn on the user's wrist. The tube attached to the cuff is connected to a syringe that acts as a chamber and the pressure sensor. The cuff is inflated and contracted through the syringe pump activated by the driver motor, and the pressure sensor starts to measure the changes in pressure. The battery has $3.7 \mathrm{~V}$ and $750 \mathrm{mAh}$ output and can be charged using an 8pin USB (Battery Model: MP952238P). The prototype's main processor Arduino Nano is connected to the PC through a USB port. The final prototype is shown below. Figure 4 depicts the actual prototype of ambulatory blood pressure monitoring device which is actually worn by the user. The driver motor and gearbox are attached to the push rod on the base of the syringe. The driver motor and the pressure sensor were connected to the Arduino Nano main processor. In addition, the syringe and pressure sensor were connected to the wristband by a Teflon tube to control the cuff and be able to measure the bio-signal from the radial artery.

\subsection{Time series ambulatory blood pressure data analysis model}

Previous studies on bio-signal data analysis used a lot of machine learning-based clustering algorithms, and there are continuous efforts on the studies of using deep-learning technologies [13-20]. This paper proposes a method for feature extraction and similarity analysis to apply a lightweight and accurate method in real-time processing.

This paper aims to develop an ambulatory blood pressure data analysis model based on supervised and unsupervised learning, and to design a time series ambulatory blood pressure data pattern analysis model for $24 \mathrm{~h}$. The 


\section{ABPM wrist tonometry waveform}

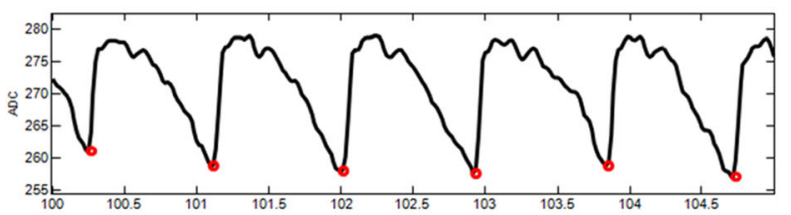

\section{Finometer finger pressure waveform}

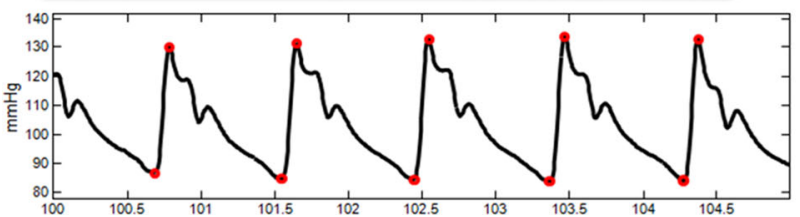

Data acquisition

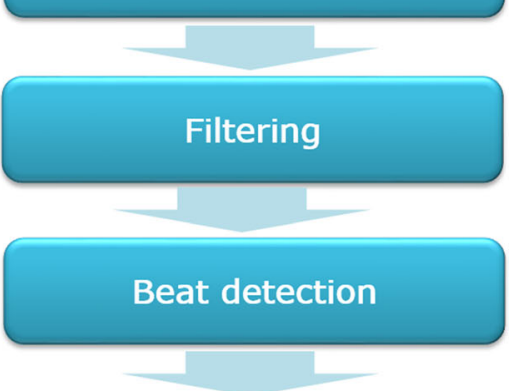

Regressive modeling

Fig. 3 Extraction process of ABPM

Fig. 4 Prototype of ABPM device

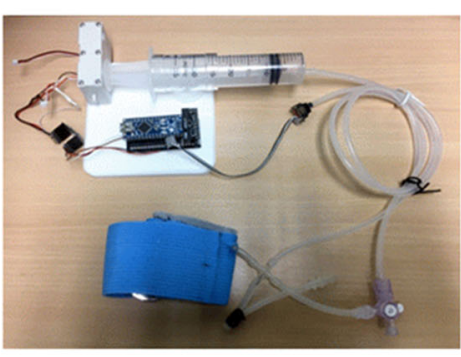

Prototype result

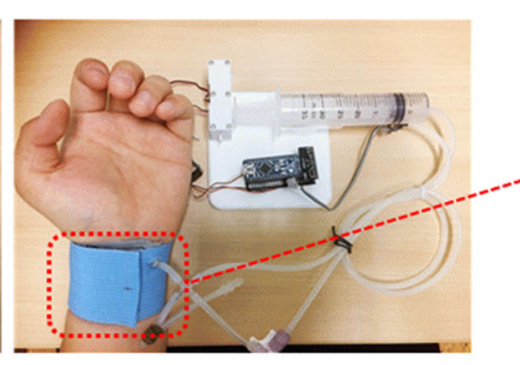

Wearing status

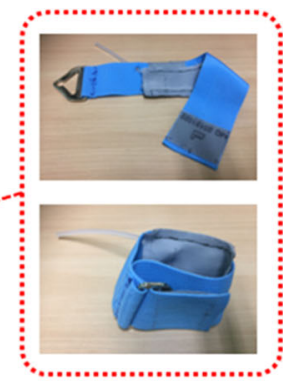

Cuff Band existing machine learning model according to the data type was applied in order to analyze the ambulatory blood pressure data pattern of the user. In general, the normal and abnormal blood pressure classification based on TOAST was defined through logistic regression analysis, and only the preliminary work for the accuracy analysis of the existing five levels (LAA, SVO, CE, UD, OD) was determined. Although the five levels for the detailed determination of blood pressure status can be identified, the normality and abnormality status of TOAST were only the primary factor for the determination of the user's blood pressure status. Thus, this paper determines only the normality and abnormality of blood pressure.

Moreover, a data mining model that combines logistic regression analysis and dynamic time wrapping (DTW) was developed suitable for similarity calculation for classification of 0 and 1 for TOAST classification. The ambulatory blood pressure data analysis process for TOAST classification is depicted in Fig. 5.

For TOAST classification, the blood pressure value (contraction, relaxation) based on RR-interval served as input to analyze the patterns through regression analysis. The similarity between analyzed pattern and reference value was calculated, and the weight of measured contraction and relaxation value was reflected to develop a model that classifies the final TOAST class.

\subsubsection{Ambulatory blood pressure data analysis modeling}

Logistic regression was applied to extract criteria curves for pattern analysis of class 0 and 1 . This method was used to predict the probability of occurrence of an event (probability) using a linear combination of independent variables. It is similar to regression and discriminant analysis in such a way that the linear combination of independent variables describes the dependent variable which was a nominal measure of binary data.

There are $\mathrm{n}$ independent variables (continuous or noncontinuous variables) and 1 dependent variable (divided non-continuous variable) which were used as dividing variables such as 0 (normal) and 1 (abnormal) and carries out a class pattern analysis applying the relevant one.

$$
\begin{aligned}
& \operatorname{Prob}(\text { Event })=\frac{e^{z}}{1+e^{z}} \\
& \mathrm{Z}=B_{0}+B_{1} X_{1}+B_{2} X_{2}+B_{3} X_{3} \cdots+B_{n} X_{n}
\end{aligned}
$$


Fig. 5 Analysis model of blood pressure data

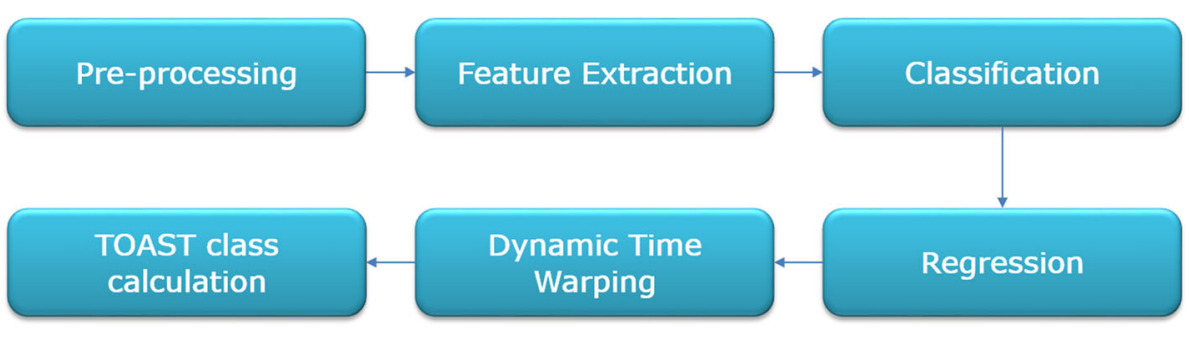

The independent variable $(X)$ changes the value of $(Z)$ wherein $(Z)$ acts as an exponent that affects the probability of event occurrence, that is, Prob (Event), so they may find what factors (independent variables, contraction/ relaxation values of ambulatory blood pressure) are risk factors of the disease (dependent variable, class 0 (normal), 1 (abnormal), and how much they affect (odds ratio). Table 2 represents the definitions of the variables described earlier. Continuous variables, non-continuous variables, and divided non-continuous variables are defined as table contents.

\subsubsection{Calculation of similarity of TOAST class patterns}

Dynamic time wrapping (DTW) technique [21-25] was used to analyze the similarity with the criteria line of the previously analyzed pattern. DTW moves in a direction that minimizes the distance between two time series, matches each other, calculates the cumulative distance from each template, and recognizes it as the minimum class. $W$ defines the mapping between each time series data $X$ and $Y$, defines the $K$ th element of $W$, calculates the corresponding maximum value, and calculates the corresponding path that satisfies the three conditions of boundary condition, continuity, and forgeability. Finding the corresponding path that minimizes the sum of $W$ is a method of calculating the similarity of DTW and can be expressed by the following equation:

$\operatorname{DTW}(X, Y)=\frac{1}{K} \sqrt{\sum_{k=1}^{K} \omega_{k}}$

$D(i, j)=d\left(x_{i}, y_{i}\right)+\min \left\{\begin{array}{c}D(i-1, j-1) \\ D(i-1, j) \\ D(i, j-1)\end{array}\right\}$
$K$ is used to compensate for the corresponding paths having different lengths. The cumulative distance $\mathrm{D}$ is the value indicating the final similarity starting from 0 and the shortest path in the DTW. In this similarity calculation method, it is necessary to control the splashing value of the impulse character due to the ambulatory blood pressure measurement characteristics. The analysis on this paper attempts to solve the problem of the measurement on the corresponding path through the exponential smoothing operation when the singular value problem occurs in the measurement of the corresponding path in the DTW similarity calculation. The final class value was calculated by reflecting the weight of the final contraction and relaxation value by comparing the similarity between the previously analyzed class learning pattern and the reference value (D1: similarity of contraction data, D2: similarity of relaxation data, alpha: weight, $g$ : group).

$\sum_{g=1}^{n}\left(D 1_{g} \times \alpha_{1}+D 2_{g} \times \alpha_{1}\right)$

\section{Performance evaluation}

\subsection{Evaluation of measurement accuracy of ambulatory blood pressure monitoring prototype}

In order to evaluate the reliability of the prototype for ambulatory blood pressure measurement, a comparison with the existing blood pressure measurement device was performed. Finometer Model-1 was used as the existing continuous blood pressure comparison device. This device measures continuous blood pressure through finger cuff pressure. Figure 6 shows the evaluation concept for evaluating the reliability of blood pressure measurement.
Table 2 Definition of variables

$$
\text { Independent variables Continuous variables }
$$

Dependent variables
Non-continuous variables

Divided non-continuous variables
Ambulatory blood pressure: Contraction Ambulatory blood pressure: Relaxation

Sex, magnitude

TOAST class

(Normal: 0, abnormal: 1) 


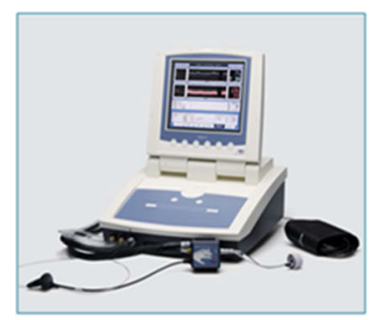
equipment

$\checkmark$ Finometer Model-1 (Finapres Medical System, The Netherlans)

$\checkmark$ blood pressure measurement

through finger cuff pressure
- Blood pressure comparison target

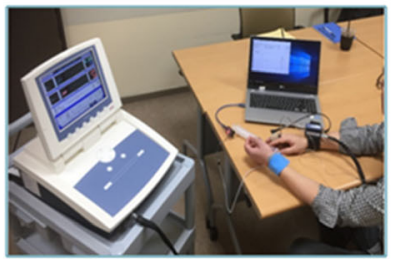

Simultaneous Data Collection Experiment

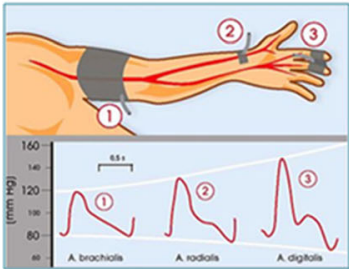

$\checkmark$ Compare waveforms after simultaneous data measurements $\checkmark$ Blood pressure estimation through Finometer data matching processing

Fig. 6 Concept of performance evaluation

Table 3 Sample of sensing data

\begin{tabular}{llllll}
\hline \multirow{2}{*}{ TEST case } & \multicolumn{2}{l}{ Finometer Model-1 sensing } & & \multicolumn{2}{l}{ Proposed device sensing } \\
\cline { 2 - 3 } & Contraction average & Relaxation average & & Contraction average & Relaxation average \\
\hline 001 & 150 & 94 & 145 & 85 \\
002 & 150 & 102 & 148 & 90 \\
003 & 141 & 91 & 133 & 86 \\
004 & 127 & 85 & 137 & 89 \\
005 & 192 & 108 & 150 & 78 \\
006 & 120 & 81 & 125 & 85 \\
007 & 131 & 75 & 135 & 82 \\
008 & 123 & 77 & 142 & 71 \\
010 & 125 & 80 & 133 & 78 \\
\hline
\end{tabular}

Table 3 shows the sample measurements. The measurement of bio-signal values of Finometer Model-1 was conducted by a professional clinician and assumed that they were accurate. Comparative matching with the blood pressure measurement conducted with the ABPM prototype was performed using the criteria value. As a result, an error of $( \pm) 2.29 \%$ was found which is similar to the Finometer Model-1.

\subsection{Ambulatory blood pressure data analysis model results}

The ambulatory blood pressure data analysis model was applied on the blood pressure information collected through the ambulatory blood pressure measurement device to determine the abnormality on the user's blood pressure. First, the low-pass and shock filters were used to pretreat the ambulatory blood pressure and extract the features. Next, pattern analysis was performed through logistic regression analysis based on the classification of the initial clinician of the training data. Then, the final
TOAST class was classified by measuring similarity (DTW + weight).

Figure 7 shows the simultaneous comparison of the pattern results of the input contraction and relaxation blood pressure data with the input blood pressure data. The numerical results were plotted to indicate the sample results of the proposed method.

The graph in Fig. 7 shows the division into the upper group (TOAST0) and the lower group (TOAST1) wherein blue represents contraction and red represents relaxation. The green lines indicate newly input patient data where DTW with criteria line for similarity analysis was calculated. The similarity (distance value) value was reflected up to the final weight, which means that the number of the two classes $(0,1)$ was included in the lesser class. For example, in Fig. 7, 9896 is given when the value of the input signal is calculated by a green line to resemble Toast 0 . And the similarity of Toast 1 means that the value of 30,868 is close to Toast 0 with a short distance of similarity. The resulting value of Toast 0 can be confirmed by the relative position of the normal curve of blood pressure contraction and 

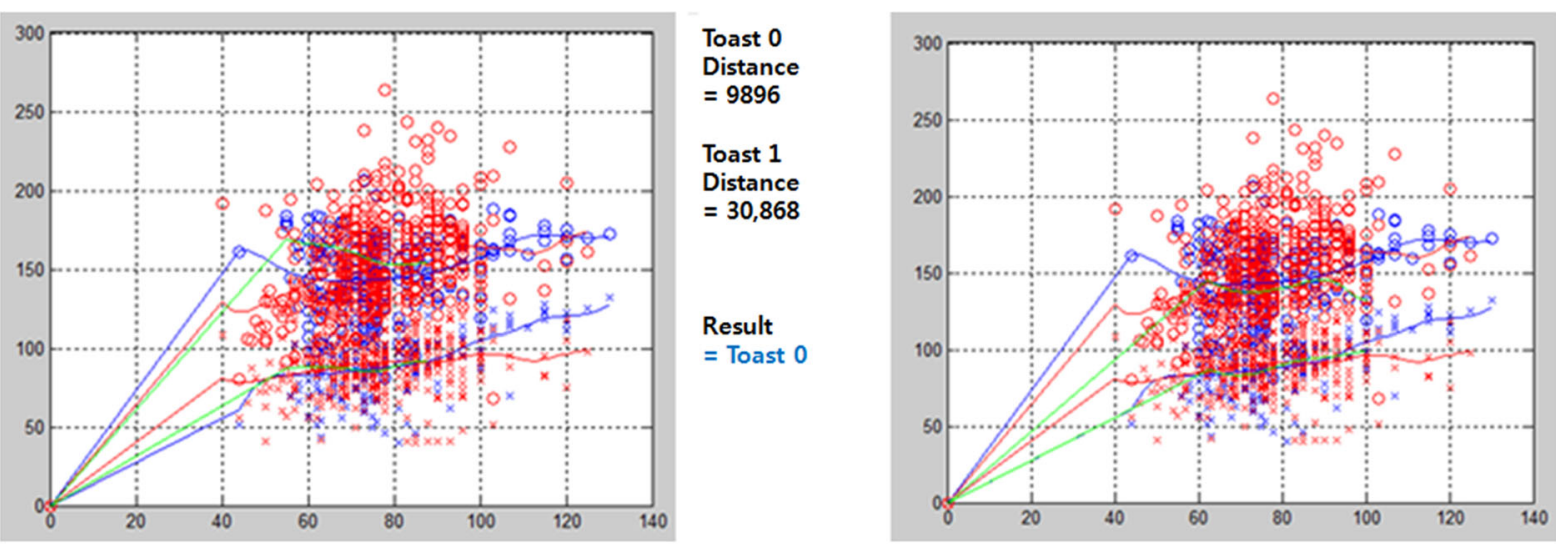

Toast 0

Distance

$=23,823$

Toast 1

Distance

$=12,608$

Result

= Toast 1

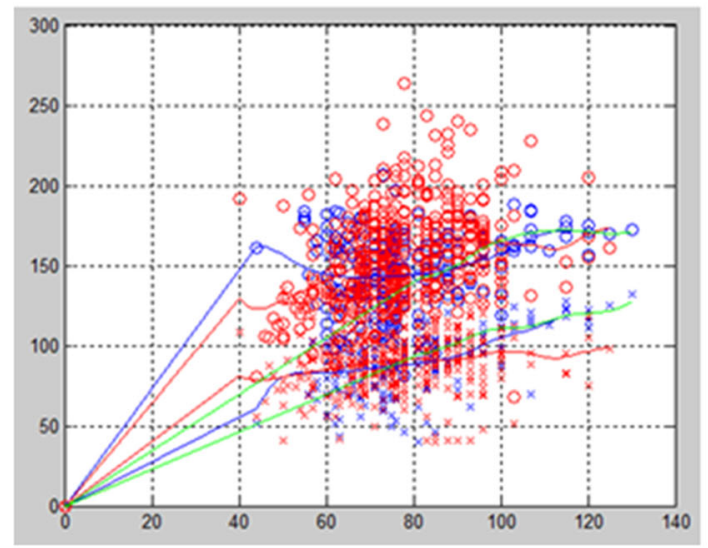

Toast 0

Distance

$=2,260$

Toast 1

Distance

$=3,763$

Result

$=$ Toast 0

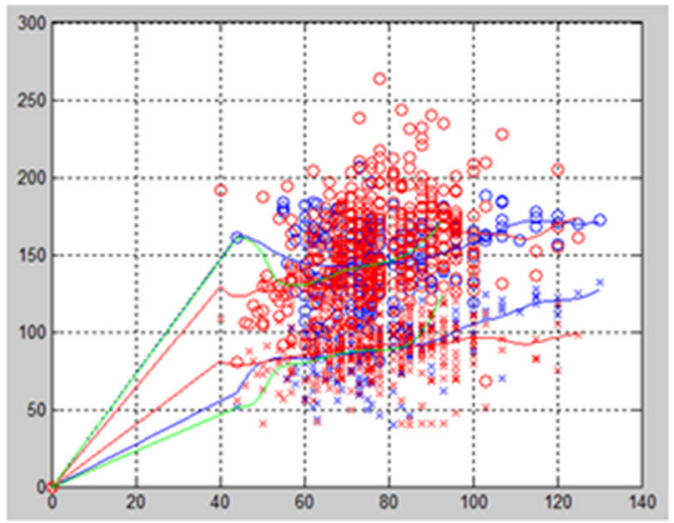

Toast 0

Distance

$=1,892$

Toast 1

Distance

$=6,593$

Result

$=$ Toast 0

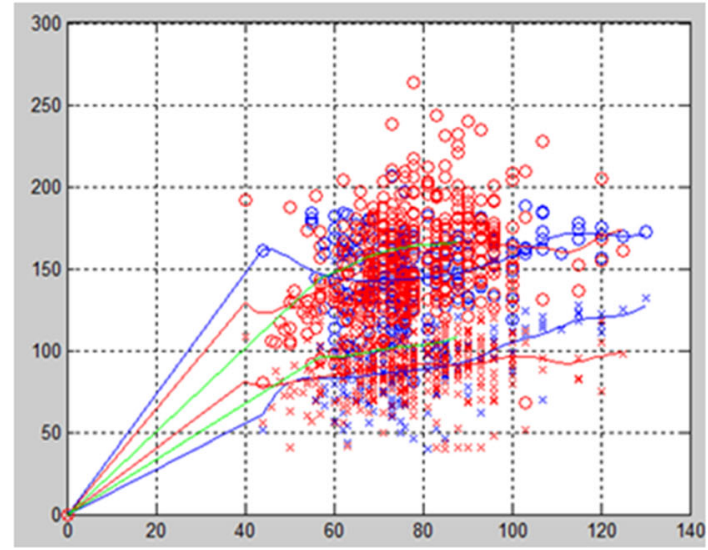

Toast 0

Distance

$=2,299$

Toast 1

Distance

$=5,330$

Result

$=$ Toast 0

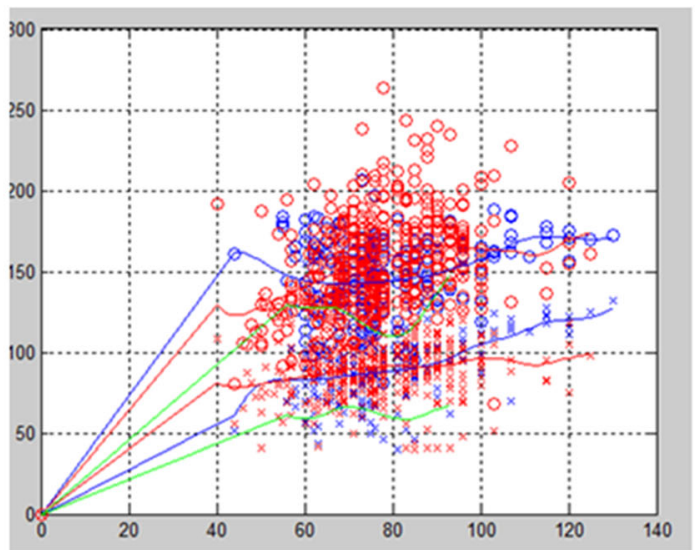

Toast 0

Distance

= 15,915

Toast 1

Distance

$=32,767$

Result

$=$ Toast 0

Fig. 7 Results of TOAST classification

relaxation. As a sample of this, six Toast matching results were shown. Table 4 shows the similarity comparison results with 20 samples using existing classification methods K-nearest neighbor (KNN) [26] and support vector machine (SVM) [27].

The results in Table 4 show the accuracy by classifying TOAST results (normal/abnormal) of each method for 20 samples (total 50 cases). Distance represents the difference of similarity with Toast's classification result. That is, the smaller the distance value, the higher the similarity. The range of distance value is indicated between 0 and 1 , the closer to 0 , the higher the accuracy is, and the closer to 0.5 , an incorrect result may have obtained. Based on the results, the proposed method could show higher accuracy than the comparison methods KNN and SVM.

The KNN method is a clustering method for simple distance differences, which results in relatively inaccurate results because it is difficult to reflect information on variance and bias. In addition, SVM method is more accurate than KNN because of the latter's inaccurate 
Table 4 Similarity comparison results

\begin{tabular}{|c|c|c|c|c|c|c|c|}
\hline \multicolumn{2}{|c|}{ Test case } & \multicolumn{2}{|c|}{ Proposed method } & \multicolumn{2}{|l|}{ KNN [26] } & \multicolumn{2}{|l|}{ SVM [27] } \\
\hline No. & Toast (N/A) & Toast (N/A) & Distance & Toast (N/A) & Distance & Toast (N/A) & Distance \\
\hline 001 & $\mathrm{~N}$ & $\mathrm{~N}$ & 0.22 & A & 0.47 & $\mathrm{~N}$ & 0.18 \\
\hline 002 & A & A & 0.40 & A & 0.32 & $\mathrm{~N}$ & 0.48 \\
\hline 003 & $\mathrm{~N}$ & $\mathrm{~N}$ & 0.11 & A & 0.44 & $\mathrm{~N}$ & 0.24 \\
\hline 004 & A & A & 0.27 & $\mathrm{~N}$ & 0.49 & A & 0.16 \\
\hline 005 & A & A & 0.12 & A & 0.24 & A & 0.17 \\
\hline 006 & $\mathrm{~N}$ & A & 0.48 & $\mathrm{~N}$ & 0.17 & $\mathrm{~N}$ & 0.47 \\
\hline 007 & $\mathrm{~N}$ & $\mathrm{~N}$ & 0.08 & $\mathrm{~N}$ & 0.33 & $\mathrm{~N}$ & 0.29 \\
\hline 008 & A & A & 0.10 & $\mathrm{~N}$ & 0.47 & A & 0.17 \\
\hline 009 & $\mathrm{~N}$ & $\mathrm{~N}$ & 0.16 & $\mathrm{~N}$ & 0.18 & $\mathrm{~N}$ & 0.19 \\
\hline 010 & A & A & 0.42 & A & 0.25 & A & 0.34 \\
\hline 011 & $\mathrm{~N}$ & $\mathrm{~N}$ & 0.15 & $\mathrm{~N}$ & 0.28 & A & 0.49 \\
\hline 012 & $\mathrm{~N}$ & $\mathrm{~N}$ & 0.11 & A & 0.46 & $\mathrm{~N}$ & 0.34 \\
\hline 013 & A & A & 0.39 & A & 0.24 & A & 0.25 \\
\hline 014 & $\mathrm{~N}$ & $\mathrm{~N}$ & 0.16 & $\mathrm{~N}$ & 0.38 & $\mathrm{~N}$ & 0.19 \\
\hline 015 & $\mathrm{~N}$ & $\mathrm{~N}$ & 0.38 & $\mathrm{~N}$ & 0.31 & $\mathrm{~N}$ & 0.12 \\
\hline 016 & A & A & 0.46 & A & 0.11 & A & 0.17 \\
\hline 017 & $\mathrm{~N}$ & $\mathrm{~N}$ & 0.42 & $\mathrm{~N}$ & 0.28 & $\mathrm{~N}$ & 0.40 \\
\hline 018 & A & A & 0.28 & A & 0.38 & $\mathrm{~N}$ & 0.48 \\
\hline 019 & $\mathrm{~N}$ & A & 0.49 & $\mathrm{~N}$ & 0.17 & $\mathrm{~N}$ & 0.13 \\
\hline 020 & A & A & 0.14 & A & 0.34 & A & 0.39 \\
\hline
\end{tabular}

results on the cluster boundary when data are mixed but shows more inaccurate results than the proposed method proposed. The proposed method does not generate boundary information for clear cluster classification but generates a representative major line for data distribution and calculates the difference between the distance and DTW to determine the TOAST. Thus, it can be verified that the proposed method yielded relatively high accuracy.

For further verification of the proposed analytical model, the confusion matrix was calculated and compared with the existing classification methods to calculate specificity, sensitivity, and accuracy. The performance evaluation verified the robustness of the proposed method. The comparison method was similarly performed with KNN and SVM.

Two methods with different points of view, such as distance between classes and hyperplane calculation method, were selected, and the results are shown in Table 5 and depicted in Fig. 8.

The error matrix value was calculated as the value of 50 cases, and the existing SVM had a high dependency on the data quality and quantity due to problems such as inaccuracy as the amount of data increases and the complexity of calculation that induces multidimensional access costs. Also, the method through KNN produced the worst result, which seems to be because only the information about the difference in distance is reflected. This seems to have been
Table 5 Confusion matrix results

\begin{tabular}{lccc}
\hline Confusion matrix & Proposed method & KNN & SVM \\
\hline True positive & 44 & 21 & 41 \\
False positive & 6 & 29 & 9 \\
True negative & 48 & 40 & 44 \\
False negative & 2 & 10 & 6 \\
\hline
\end{tabular}

calculated without additional information such as bias information. In contrast, the proposed method yielded a relatively high performance. The proposed ambulatory blood pressure analysis model provides a learning-based algorithm to minimize user intervention. Among the aforementioned sample results, errors in the similarity calculation for TOAST recognition occurred when the information on the normalization curve was incorrect in the contraction and relaxation data in the primary case of recognition error. This is caused by impulse noise in preprocessing and is thought to be solved by adding filtering.

\section{Conclusion}

Ambulatory blood pressure monitoring measures and records blood pressure several times during normal activity and has several advantages over the fractional blood 


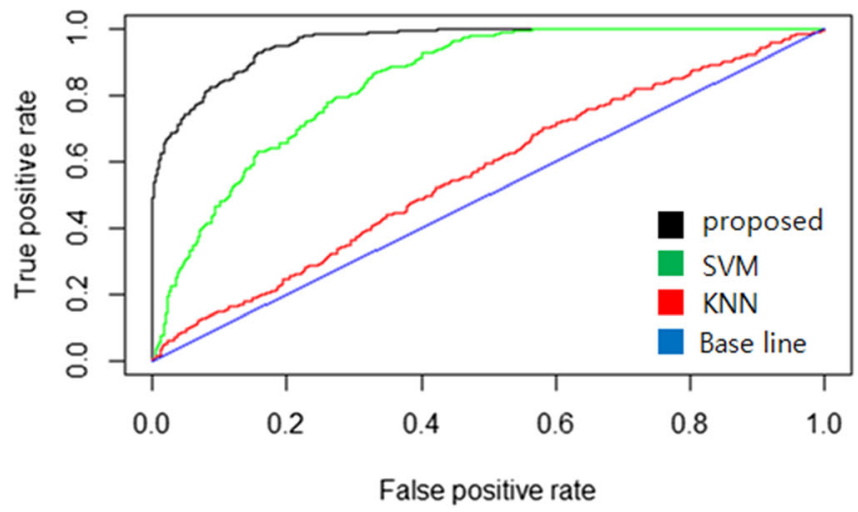

Fig. 8 Results of ROC curve and accuracy

pressure measured in the hospital. With the development of technology, the small size and simple usage allow ease on handling devices at home, creating new demand, and in fact, various automatic blood pressure monitors have been sold and distributed such that the scale of blood pressure monitor market is very wide. The ambulatory blood pressure monitoring device which is capable of measuring fragmentary blood pressure has more advantages in managing hypertension since it can provide information on fluctuations of blood pressure patterns even though it is inconvenient to use and less portable.

This paper deals with the development of an ambulatory blood pressure device and intelligent healthcare management system that can continuously manage the ambulatory blood pressure of users. A prototype of tonometric wristworn ambulatory blood pressure monitoring device was developed which is highly portable, easy to wear, and capable of improving the reliability of measured bio-signals. The measured blood pressure values were compared with the existing popular equipment in order to verify the reliability of the developed prototype. And the proposed analysis method was found to be more effective through the comparison analysis with the existing classification methods. Finally, the health status of hypertension patients can be evaluated for a limited time. In addition, if there was a lot of impulse noise in the shrinkage, loosening data, the inaccuracy of the normalization curve generation could be identified. This caused a problem with the TOAST class judgment accuracy, but I think it will be improved if some additional filtering work for the preprocessing work is reflected. Filtering can take into account a variety of signal improvement filters, such as the median filter [28] or the low-pass filter [29], which eliminates impulse-based noise. In the future, as the concept of preventive healthcare is essentially needed, an additional study using deep learningbased prediction technology $[30,31]$ will be carried out.

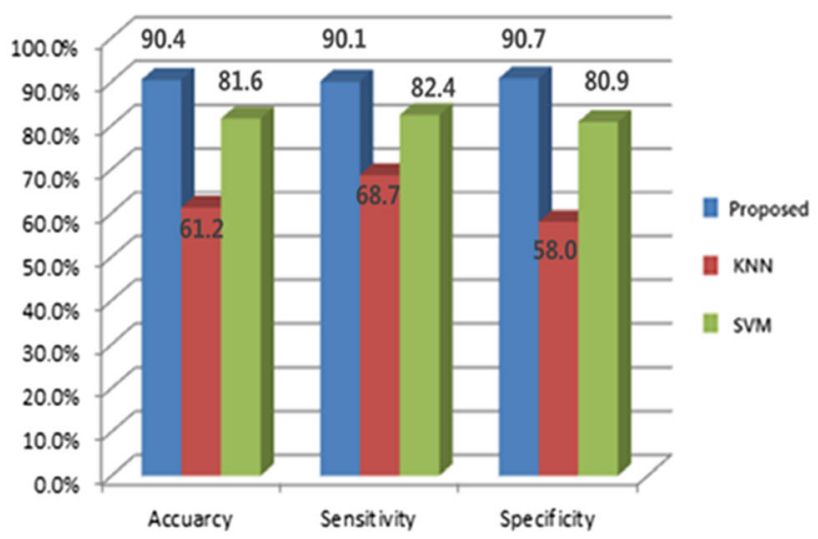

Acknowledgement This work was supported by information \& communications Technology Promotion (IITP) grant funded by the Korea government (MSIT) (2019-0-00225, AI Convergence Leading Project).

Funding No fund/grant support relevant to this article was reported.

Data availability The sensing data used to support the findings of this study are included within the article.

\section{Compliance with ethical standards}

Conflict of interest The authors declare that there are no conflicts of interest regarding the publication of this paper.

Open Access This article is licensed under a Creative Commons Attribution 4.0 International License, which permits use, sharing, adaptation, distribution and reproduction in any medium or format, as long as you give appropriate credit to the original author(s) and the source, provide a link to the Creative Commons licence, and indicate if changes were made. The images or other third party material in this article are included in the article's Creative Commons licence, unless indicated otherwise in a credit line to the material. If material is not included in the article's Creative Commons licence and your intended use is not permitted by statutory regulation or exceeds the permitted use, you will need to obtain permission directly from the copyright holder. To view a copy of this licence, visit http://creativecommons. org/licenses/by/4.0/.

\section{References}

1. O'Brien E, Atkins N, Staessen J (1995) State of the market: a review of ambulatory blood pressure monitoring devices. Hypertension 26(5):835-842

2. O'Brien E (2001) State of the market for devices for blood pressure measurement. Blood Pressure Monit 6(6):281-286

3. Lawes CMM, Hoorn SV, Rodgers A (2008) Global burden of blood-pressure-related disease, 2001. Lancet 371(9623): 1513-1518

4. Groppelli A et al (1992) Evaluation of noninvasive blood pressure monitoring devices Spacelabs 90202 and 90207 versus resting and ambulatory 24-hour intra-arterial blood pressure. Hypertension 20(2):227-232 
5. McGrath BP (2002) Ambulatory blood pressure monitoring. Med J Aust 176(12):588-592

6. O'Brien E et al (2013) European society of hypertension position paper on ambulatory blood pressure monitoring. J Hypertens 31(9):1731-1768

7. O'brien E et al (2003) European society of hypertension recommendations for conventional, ambulatory and home blood pressure measurement. J Hypertens 21(5):821-848

8. White WB et al (1993) National standard for measurement of resting and ambulatory blood pressures with automated sphygmomanometers. Hypertension 21(4):504-509

9. O'Brien E et al (2001) Blood pressure measuring devices: recommendations of the European Society of Hypertension. BMJ 322(7285):531-536

10. O'Brien E et al (2000) Use and interpretation of ambulatory blood pressure monitoring: recommendations of the British Hypertension Society. BMJ 320(7242):1128-1134

11. Eun S-J, Kim J-Y, Lee SH (2019) Development of customized diving computer based on wearable sensor for marine safety. IEEE Access 7:17951-17957

12. Whangbo T-K et al (2018) Personalized urination activity recognition based on a recurrent neural network using smart band. Int Neurourol J 22(2):S91

13. Wang J, Gao Y, Wang K, Sangaiah AK, Lim S-J (2019) An affinity propagation based self-adaptive clustering method for wireless sensor networks. Sensors 19(11):2579. https://doi.org/ $10.3390 / \mathrm{s} 19112579$

14. Wang J, Gao Y, Liu W, Sangaiah AK, Kim H-J (2019) An improved routing schema with special clustering using PSO algorithm for heterogeneous wireless sensor network. Sensors. https://doi.org/10.3390/s19030671

15. Wang J, Gao Y, Liu W, Wu W, Lim S-J (2019) An asynchronous clustering and mobile data gathering schema based on timer mechanism in wireless sensor networks. Comput Mater Continua 58(3):711-725

16. Wang J, Cao J, Ji S, Park JH (2017) Energy efficient clusterbased dynamic routes adjustment approach for wireless sensor networks with mobile sinks. J Supercomput 73(7):3277-3290

17. Wang J, Gao Y, Yin X, Li F, Kim H-J (2018) An enhanced PEGASIS algorithm with mobile sink support for wireless sensor networks. Wirel Commun Mob Comput 2018, 9472075

18. Mancia G et al (1997) Ambulatory blood pressure is superior to clinic blood pressure in predicting treatment-induced regression of left ventricular hypertrophy. Circulation 95(6):1464-1470
19. Li Y et al (2006) Ambulatory arterial stiffness index derived from 24-hour ambulatory blood pressure monitoring. Hypertension 47(3):359-364

20. Perloff D, Sokolow M, Cowan R (1983) The prognostic value of ambulatory blood pressures. JAMA 249(20):2792-2798

21. Muda L, Begam M, Elamvazuthi I (2010) Voice recognition algorithms using mel frequency cepstral coefficient (MFCC) and dynamic time warping (DTW) techniques. arXiv:1003.4083

22. Dhingra SD, Nijhawan G, Pandit P (2013) Isolated speech recognition using MFCC and DTW. Int J Adv Res Electr Electron Instrum Eng 2(8):4085-4092

23. Lichtenauer JF, Hendriks EA, Reinders MJT (2008) Sign language recognition by combining statistical DTW and independent classification. IEEE Trans Pattern Anal Mach Intell 30(11):2040-2046

24. Pravdova V, Walczak B, Massart DL (2002) A comparison of two algorithms for warping of analytical signals. Anal Chim Acta 456(1):77-92

25. Faundez-Zanuy M (2007) On-line signature recognition based on VQ-DTW. Pattern Recogn 40(3):981-992

26. Zhang $S$ et al (2017) Efficient knn classification with different numbers of nearest neighbors. IEEE Trans Neural Netw Learn Syst 29(5): 1774-1785

27. Zhang T et al (2006) Fall detection by wearable sensor and oneclass SVM algorithm. In: Intelligent computing in signal processing and pattern recognition. Springer, Berlin, pp 858-863

28. Esakkirajan $\mathrm{S}$ et al (2011) Removal of high density salt and pepper noise through modified decision based unsymmetric trimmed median filter. IEEE Signal Process Lett 18(5):287-290

29. Ahn D et al (2001) A design of the low-pass filter using the novel microstrip defected ground structure. IEEE Trans Microw Theory Tech 49(1):86-93

30. Su P et al (2018) Long-term blood pressure prediction with deep recurrent neural networks. In: 2018 IEEE EMBS international conference on biomedical \& health informatics (BHI). IEEE, 2018

31. Li X, Wu S, Wang L (2017) Blood pressure prediction via recurrent models with contextual layer. In: Proceedings of the 26th international conference on world wide web. International World Wide Web Conferences Steering Committee

Publisher's Note Springer Nature remains neutral with regard to jurisdictional claims in published maps and institutional affiliations. 